\title{
PROBLEMAS TERAPEUTICOS EN ALGUNOS PROCESOS TUBERCULOSOS DE LA INFANCIA
}

\author{
DIS. JORGE PENA CERECEDA Y HUMBERTO PINTO \\ Hospital "Manael Arriarán".
}

\section{A. - PRIMO INFECCIón TUBERCULOSA}

1) Evolución espontánea de la primo. infección Tbc. $y$ planteamiento terapéutico. - La evolución de la primoinfección Tbc. es extraordinariamente variable de uno a otro caso $y$, en líneas generales, podría decirse que se influye por la edad del paciente, condición económico-social, calidad del ambiente (posibilidad de reinfección) o curso evolutivo mismo de la enfermedad. Todos estos factores deben tenerse presente cuando se plantea la formulación de un tratamiento a base de antibióticos (estreptomicina generalmente asociada a otra droga) para controlar en forma más efectiva el curso de la primoinfección. Asimismo las circunstancias ya señaladas deben considerarse en la avaluación de los resultados terapéuticos obtenidos con un determinado tipo de tratamiento.

Existen quienes (E. Lincoln) manifiestamente no se muestran partidarios del tratamiento de la primoinfección con antibióticss, aduciendo argumentos variados (ineficacia relativa de las drogas utilizadas en este tipo de procesos, carácter espontáneo de la evolución en muchos casos, posibilidad de crearse estreptomicinoresistencia). Sin desconocer el valor de algunos de los planteamientos expuestos, debe tenerse presente que en nuestro medio la primoinfección puede verse influenciada desfavorablemente por la desnutrición concomitante, la imposibilidad de recurrir a los beneficios de la cura climática o sobrealimentación y la existencia a veces de contagio en el ambiente familiar. En el lactante estos factores alcanzan, como es sabido, especial relieve.

Es útil ilustrar al respecto con lo que ha ocurrido en nuestra experiencia, en el control de un material numéricamente importante de primoinfección tuberculosa 1 .911 niños atendidos en el Consejo de Defensa del Niño) entre 1938 y 1950 . Se trató de pacientes generalmente escolares 11.711 casos) que inicialmente presentaban adenopatías tráqueo-brónquicas no complicadas y que no recibieron antibióticos (estreptomicina). En la evolución de estos enfermos pudo apreciarse que en
151 casos existieron complicaciones, cuyo mayor número ocurrió dentro de los 4 primeros años de control; en este período predominaron las complicaciones de tipo diseminado (miliar, meningitis Tbc., Tbc. broncógena o abdominal). A partir de esta época hubo mayor incidencia de complicaciones de carácter localizado cadenitis cervical, conjuntivitis flictenular, pleuritis, atelectasia). Entre los 9 y 10 años, aparece escaso número de complicaciones de reinfección. Se desprende, en suma, que dentro de los primeros cuatro años de evolución de una primoinfección Tbc., hay alguna tendencia a la aparición de complicaciones que implican un pronóstico a veces grave, por lo que en estos casos podría aparecer indicada Ia administración de antibióticos. Las complicaciones aparecidas posteriormente en el curso del periodo escolar si bien no tiene la gravedad manifiesta de las que se advierten con anterioridad, también pueden exponer a secuelas de tratamiento difícil (bronquiectasia, retracción torácica). Las infiltraciones de la edad puberal, finalmente, por su carácter progresivo, también permitirían aconsejar la administración de estreptomicina.

2) Experiencia en el tratamiento de la primoinfección Tbc. - En nuestra experiencia (78 niños, la mitad aproximadamente recibió estreptomicina en un plazo de cerca de 4 meses) hubo menor número de complicaciones $(5$ y 15 en los controles y menor duración de la actividad de las lesiones $(3$ a 6 meses en los tratados ! cuando los pacientes recibieron tratamiento estreptomicínico. Con estos antecedentes, creemos que es aconsejable tratar la primoinfección Tbc. en el niño menor (lactancia) en cualquiera de sus formas. La primoinfección evolutiva del niño mayor también requiere del tratamiento antibiótico. En ciertas circunstancias, factores extrínsecos (condición económico-social fundamentalmente) pueden justificar un tratamiento quimioterápico.

Puede recomendarse como útil una pauta terapéutica, que consulte la administración de estreptamicina ( $1: 2$ o 1 gramo según edad dos veces por semana $\mathrm{y}$ durante un plazo de tres meses, por el in- 
conveniente de la resistencia a la droga (a Jos 42 días hay $35 \%$ de cepas resistentes y a los 120 días, un $75 \%$. La asociación al PAS ino siempre bien tolerado, 5 a 10 gramos, en 3 dosis) o la hidrazida (10 miligramos por $\mathrm{Kg}$. en dos dosis) es una norma adecuada, para prevenir la resistencia, pudiendo prolongarse este tratamiento durante seis o más meses, de acuerdo a la regresión de las lesiones que se compruebe.

\section{B. - MENINGItis tUbERCUlOSA}

a) Antecedens generales de orden clinico. - En sentido estricto en la mayoría de los casos, la meningitis tuberculosa se acompaña de lesiones encefálicas de diversos tipos y que pueden tener traducción clínica, en estas ocasiones la enfermedad corresponde conceptualmente $y$ con mayor propiedad a una meningo-encefalitis. Es importante tener presente este punto de vista, ya que en la evolución actual de la afección que se somete a tratamiento específico, es posible advertir variadas manifestaciones de tipo neurológico, que obedecen muchas veces a daño encefálico, ajeno al componente mismo meníngeo de la enfermedad, que suelen persistir como fenómenos patológicos de tipo residual.

En una etapa vaanzada la meningitis tuberculosa se caracteriza por un conjunto polimorfo de sintomas, ajenos algunos de ellos al síndrome meníngeo propiamente tal. En esta fase el diagnóstico no constituye generalmente problema, pero desde el punto de vista terapéutico, para formular un tratamiento oportuno con mayor probabilidad de eficacia, es mucho más importante conocer las manifestaciones iniciales de la enfermedad (cambio de carácter, cefalea, signos de proceso infeccioso general) que pueden o no. ocurrir en un paciente en el que anteriormente se hizo el diagnóstico de infección tuberculosa. Los sintomas de probable hipertensión intracraneana (vómito fácil y repetido, cefalea intensa) ocurren a veces en forma algo tardía; en estos casos la punción lumbar resulta imperativa. Posiblemente este mismo método de explora ción (P. L.) practicado en el curso de una primoinfección activa tuberculosa, resulte de utilidad, para el despistaje precoz de la participación meníngea en una etapa que no tenga traducción clínica manifiesta.

b) Pautas terapéuticas aplicadas.-Los planes de tratamiento de la meningitis tu- berculosa han ido variando en los últimos años, de acuerdo con la aparición $\mathrm{y}$ disponibilidad de agentes farmacológicos de mayor eficacia, del conocimiento de la utilidad en su asociación y de los riesgos inherentes (toxicidad) a las mismas drogas utilizadas. Tanto el tipo de medicamento, como su dosíficación, vía de administración y plazo de empleo han sido modificados sucesivamente de acuerdo con Ja experiencia adquirida con diversos esquemas terapeuticos. Por este motivo toda avaluación terapéutica es difícil de realizar y las sugerencias generales que pueden establecerse como resultado de una investigación en un material amplio, no pueden nunca tener el carácter de definitivas.

Desde 1948 hasta el presente año, hemos tenido opcrtunidad de tratar con antibióticos y de controlar por un plazo variable (siempre mayor de dos semanas) 93 casos de meningitis tuberculosa. El tratamiento se inició en una época variable i en la primera semana en 39 casos, en 27 en la segunda semana y en 12 con posterioridad al primer mes). En 9 casos el tratamiento se realizó en forma muy precez (pacientes con meningitis serosa).

Fundamentalmente hemos realizado tratamientos sujetos a algunas variaciones en lo que respecta a calidad o dosaje del antibiótico, esquemáticamente podríamos ubicar nuestra casuística como sometida a tres esquemas terapéuticos:

a) Tratamiento corto de 2 a 3 meses, con intervalos de 15 o más días, repetido dos o tres veces. Dosis altas ( 300 mgrs.) intratecales $y$ reducidas por via paraenteral $\{37$ casos?.

b) Tratamiento continuado con dosis de 50 mgrs. (intratecal) y de 400 mgrs. a 1 gr. por via general. Punción lumbar diaria hasta la normalización del líquido céfalo-raquideo $\mathrm{y}$ posteriormente dia por medio y dos veces por semana hasta completar un año (36 casos).

c) En este esquema se utilizó hidrazida por vía oral, después de seis meses de tratamiento estreptomicínico, de duración fluctuante entre 3 y 6 meses. (Dosis de 5 mgrs. por $\mathrm{Kg}$. de hidrazida en dos dosis diarias). También en algunos enfermos la asociación a la hidrazida se realizó en forma precoz (durante 10 meses). En nuestra última casuistica hemos utilizado en algunos pacientes, la hidrazida en forma exclusiva.

Los resultados obtenidos nos revelan que la mortalidad globaI tiene alguna re- 
lación con la edad de los pacientes, sin influenciarse por la asociación a lesiones tuberculosas hematógenas y determinándose en forma Ilamativa por el tipo de pauta terapéutica preserita. (Plan A, con mortalidad de $91 \%$ en el cuarto trimestre de evolución; Plan B, con elevada mortalidad inicial, pero que se reduce posteriormente; Plan $\mathrm{C}$, con mortalidad inicial nuIa, que alcanza sólo un $5 \%$ en el sexto mes de control).

La regresión de los signos meníngeos fué total en el Plan C. $\mathrm{y}$ mucho menos eviclente en los otros dos esquemas de tratamiento. Ias modificaciones del líquido céfalo-raquídeo, al iniciar el tratamiento, consisten en elevación de la albúmina y de los elementos celulares, lo que se ha imputado a la acción de la estrejtomicina intratecal (pero también ocurre en los tratados con hidrazida oral). Ia normalización general deI liquido ocurre en el Plan B, después del sexto mes, en cambio, en los tratados con hidrazida sola o asociada a estreptomicina, esta regresión es más precoz.

Complicaciones y secuelas. - Entre las primeras deben señalarse la presencia de síndrome hidrocefálico (organización de exudados) traducido o no por síntomas clínicos (edema papilar, vómitos persistentes, cefalea) y que se despista por el neumoencefalograma ( 8 casos) o se comprueba en la autopsia. Con el Plan C, no se ha apreciado esta complicación. La mono o hemiparesia fué una complicación más frecuente entre los enfermos tratados según el esquema $\mathrm{B}$ y $\mathrm{C}, \mathrm{y}$ que seguramente no han recibido el tratamiento en forma oportuna. En la iniciación de muchos cuadros meníngeos y en probable relación con tuberculomas corticales o subcorticales, se presentó con alguna frecuencia sindrome convulsivo.

Entre las secuelas, destaca la sordera, en cuya patogenia se hace intervenir la acción tóxica de la estreptomicina, sin deseçhar a veces la influencia que puede tener el mismo daño encefálico en la génesis de esta alteración. En 3 casos se comprobaron fracturas espontáneas secundarias a lesiones tróficas. A veces se comprueban, con posterioridad al tratamiento, alteraciones psíauicas, de grado variable $y$ aue en un enfermo presentó las características propias de la locura moral. EI estudio electroencefalográfico reveló en la mitad de los enfermos estudiados 19 de 18 ) anormalidad, que en 4 pacientes se caracterizó como de tipo difuso. En casi todos los enfermos con electroencefalograma alterado, existía compromiso neurológico desde la iniciación del tratamiento.

Recaídas. - Constituyen uno de los problemas terapéuticos más complejos; son más frecuentes entre enfermos tratados con los planes A y B y no se comprobaron con el esquema terapéutico $\mathrm{C}$. Ocurren generalmente entre los 6 y 8 meses de tratamiento, pero pueden aparecer a veces mucho más tarde ( 2 casos más allá del primer añol. En el curso de las recaidas, se comprueba el fallecimiento de la mayor parte de los pacientes trata. dos con el Plan $\mathbf{A}$, de menor número de casos que siguieron el Plan $\mathrm{B}, \mathrm{y}$ de un paciente tratado según el esquema $\mathrm{C}$.

Periodo de control. - Sobreviven 3 pacientes que siguieron el Plan A y que cuentan con 2, 3 y 5 años de observación. Del Plan B, sobreviven 24 enfermos 12 con 3 años de observación, 9 con 2 y medio años, 10 con 2 años y 3 con más de 1 año). De los 20 casos del Plan C, sólo fallece uno a los seis meses de tratamiento $\mathrm{y}$ los sobrevivientes tienen algo más de un año de observación.

Síntesis terapéatica $y$ pauta de tratamiento aconsejable. -.. En el estado actual de nuestra experiencia, sugerimos que un tratamiento oportuno de la meningitis tuberculosa, para alcanzar eficacia, debe basarse en la asociación medicamentosa (estreptomicina, PAS e hidrazida) prolongada durante un período prudencial. Las premisas fundamentales de un esquema terapéutico aconsejable residen en:

a) Estreptomicina intramuscular i una inyección diaria de $500 \mathrm{mgrs}$. o $1 \mathrm{gr}$., según edad) durante un plazo fluctuante entre 6 y 10 meses. Podrá no ser indispensable la administración diaria de la estreptomicina e inyectarse cada 3 dias, en los casos de favorable evolución clínica y manifiesta regresión del líquido céfalo-raquídeo.

b) Hidrazida por vía oral. a la dosis de 10 mgrs. por $\mathrm{Kg}$., por un plazo similar al seguido con la terapéutica general a base de estreptomicina.

c) Estrepto-PAS intratecal ientre 40 y 80 mgrs., según edad) diariamente durante los 3 primeros meses y cada 307 dias, con posterioridad de acuerdo a la evolución clínica y regresión de las alteraciones del líquido céfalo-raquíden. Es posible que la exclusión de la vía intratecal, no resulte desfavorable, como hemos comprobado en una casujstica reducida. 